$\mathbb{T}$ periodica polytechnica

Chemical Engineering

$5 6 / 1 ( 2 0 1 2 ) 2 1 \longdiv { 2 9 }$

doi: $10.3311 /$ pp.ch.2012-1.03

web: http://www.pp.bme.hu/ch

(c) Periodica Polytechnica 2012

RESEARCH ARTICLE

\section{Processing sweet sorghum into bioethanol - an integrated approach}

\author{
Miklós Gyalai-Korpos / Tünde Fülöp / Bálint Sipos / Katalin Réczey
}

Received 2011-11-30, accepted 2012-02-09

\begin{abstract}
Numerous evidences have been provided that juice of sweet sorghum and the leftover after squeezing, the bagasse can be a proper feedstock for bioethanol production. The possibility to integrate a side stream of sweet sorghum processing into the biomass-to-ethanol process was investigated in this study. The liquid fraction, a side stream of the necessary pretreatment of the bagasse was utilized as carbon source for Trichoderma reesei RUT-C30 to produce cellulase enzymes for biomass conversion. However, to overcome the inhibitory nature of the liquid fraction, pre-adaptation of the fungus on solid media was carried out previous to submerged fermentations. The results show that with this approach the lag phase caused by the inhibitors could be markedly shortened and an enhancement of the final enzyme production could be achieved when comparing the preadapted strains to reference.
\end{abstract}

\section{Keywords}

sweet sorghum - steam pretreatment . Trichoderma reesei . cellulase $\cdot$ bioethanol

\section{Acknowledgement}

The Hungarian National Research Fund (OTKA - K 72710) and the New Hungary Development Plan (Project ID: TÁMOP4.2.1/B-09/1/KMR-2010-0002) are kindly acknowledged for their financial support. Guido Zacchi is gratefully acknowledged for letting use the steam pretreatment unit.

\section{Miklós Gyalai-Korpos}

Department of Applied Biotechnology and Food Science, BME, Budapest H1111 Szent Gellért tér 4, Hungary

e-mail: miklos_gyalai-korpos@mkt.bme.hu

Tünde Fülöp

Bálint Sipos

Katalin Réczey

Department of Applied Biotechnology and Food Science, BME, Budapest H1111 Szent Gellért tér 4, Hungary

\section{Enhanced utilization of sweet sorghum in bioethanol production by adaptation of Trichoderma reesei RUT- C30}

\section{Background}

Different cultivars of sweet sorghum (Sorghum saccharatum), especially the ones that have been bred in Hungary and adapted to the local environmental conditions, can be viable solutions for rural fuel supply. Sweet sorghum is a sugar cane-like plant, containing juice with high concentration of sucrose in the stem that can be effectively extracted by squeezing and thereafter readily fermented to ethanol by baker's yeast. The leftover, like in case of sugar cane processing, is called bagasse. In contrast to sugar cane, sweet sorghum can be grown on continental climate, however with only one harvest per year - the frost terminates the growth of the plant. In this case the cultivation period is between April and mid September-October when the sugar content is the highest. Advantages of sweet sorghum cultivation in Hungary are the high sugar yield (5-6 t/ha), the drought tolerance (no irrigation is needed) and the modest demand for soil (that are not appropriate for corn or wheat). With these properties the cultivation of sweet sorghum will not face the food versus fuel debate often related to bioethanol production.

Feasibility of ethanol production from sweet sorghum juice has already been presented in many studies [1] furthermore several attempts have also been made to use the bagasse for second generation ethanol production [7, 19,21]. The technological difficulty of sweet sorghum processing is the short harvest period making the juice available only for 1-2 months in the year: the juice cannot be stored because the microbes including its natural microbial flora are degrading the easily fermentable sugar content.

The utilization of the bagasse and any other lignocellulosic by-product could balance the annual short availability of the juice. A theoretical scheme for the integrated sweet sorghum to ethanol process is demonstrated on Fig. 1. This process is capable to utilize the whole plant as well as other lignocellulose based residues for ethanol production all year round. This would mean a great opportunity for the integration of first and second generation technologies as well as to balance the main disadvan- 


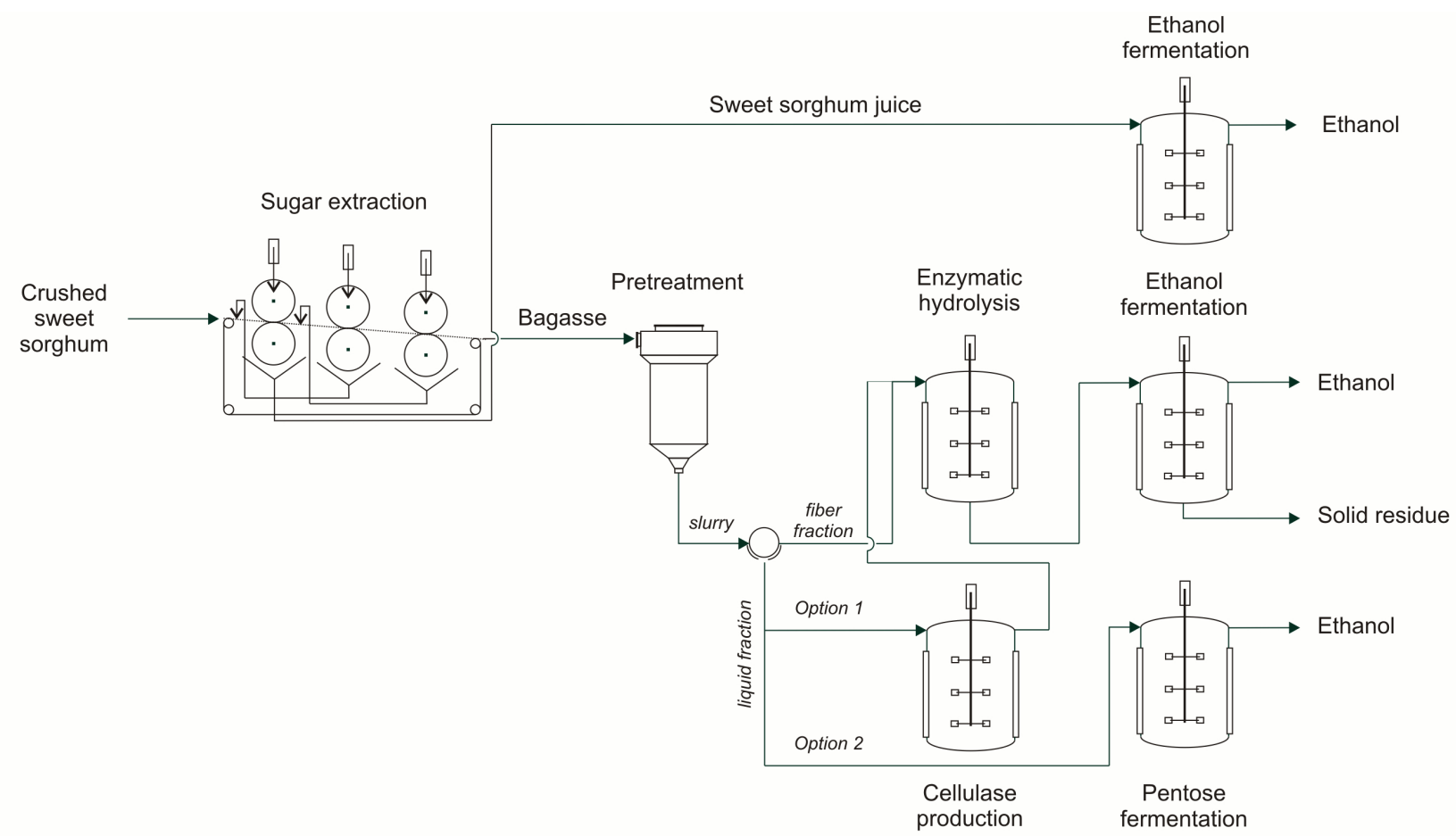

Fig. 1. Scheme of integrated sweet sorghum processing into ethanol, adapted from Sipos, et al. [21]

tage of the utilization of the juice for ethanol production.

Contrarily to first generation ethanol production that uses starch or sucrose containing feedstock, second generation ethanol production is based on lignocellulosic biomass [11]. However, because of the more resistant structure of lignocellulosic materials, the enzymatic hydrolysis must be preceded by pretreatment that aims to set cellulose chains free of the lignin matrix to be available for enzymes and eliminate the other component of lignocelluloses, namely the hemicelluloses (composed mostly of pentoses) in a soluble form [2]. The pretreatment is often a physical-chemical process, like steam explosion that applies severe pressure and temperature conditions for an exact time which after the pressure is rapidly expanded to atmospheric causing destruction in the lignocellulosic structure. Due to the severe conditions the breakage of the useful sugar molecules is also expected creating soluble compounds with inhibitory effect on microbial growth [14]. After pretreatment the slurry is separated to the cellulose containing fiber fraction subsequently exposed to enzymatic hydrolysis and the liquid fraction containing inhibitory compounds making the further utilization difficult. Despite of this difficulty the utilization of liquid fraction attains interest because of its high sugar content mostly in forms of pentoses available on-site. However, before any kind of utilization the liquid fraction most likely needs to be detoxified to decrease its inhibitory property. Chandel and coworkers [4] recently delivered a review on the possibilities to overcome inhibition covering several physical, chemical and biological methods. There are two possible utilizations of the liquid fraction that could be integrated into the biomass-to-ethanol process. One is the utilization of it by different microbes, mostly by ethanologenic bacteria and yeast strains in a separate fermentation step to increase the overall ethanol yield. Extensive work has been done on developing inhibitor tolerant and pentose utilizing yeast strains [5, 9]. The other option is to use this fraction for on-site cellulase production by Trichoderma reesei [8,20, 23]. However, these efforts with Trichoderma have mostly failed when the total concentration of the inhibitors exceeded the level of tolerance of T. reesei.

The effect of acetic acid, one of the compounds present in the liquid fraction, on cellulase production by $T$. reesei RUT C30 was investigated up to $3 \mathrm{~g} / \mathrm{L}$ on washed steam pretreated willow as carbon source at $\mathrm{pH} 6.0$ and no inhibitory effect was found (FPA of 1.3 FPU/mL was reached on day 7). However, when both furfural and acetic acid were added into the medium a clear inhibitory effect was observed. Interestingly, it was also found that the acetic acid at low concentrations appeared to reduce the inhibitory effect of the furfural [24]. Similar effect was observed with the liquid fraction of steam pretreated wheat straw. T. reese $i$ was not able to utilize the liquid fraction without detoxification, even though after detoxification the total concentration of inhibitors was the same with eliminating furfural and 5-hydroxymethyl furfural (HMF) but increasing the concentration of aliphatic acids [8]. This explains well that the synergies between compounds with possible inhibitory effect may be more relevant than their discrete concentration. Many sources report that $T$. reese $i$ is able to consume several inhibitors when concentrations are below the inhibitory level [8, 12, 17, 24].

These findings indicate that $T$. reesei possesses a relative resistance to inhibitors that prompted us to find a biological way to enhance this property. This study concentrates on on-site cellulase enzyme production using the liquid fraction as carbon source that could be easily and at low cost integrated into the 
process to overcome the barriers related to second generation ethanol production. As other parts of the process have been demonstrated earlier to be feasible [7, 19,21], neither the ethanol fermentation of juice nor the enzymatic hydrolysis of pretreated bagasse were investigated this time.

\section{Materials and methods}

\section{Strain and raw materials}

For enzyme production experiments $T$. reesei RUT C30 (ATCC56765) strain purchased from the American Type Culture Collection was used. It was maintained on malt agar slants at $30^{\circ} \mathrm{C}$ composed of $20 \mathrm{~g} / \mathrm{L}$ malt extract, $20 \mathrm{~g} / \mathrm{L}$ agar, $5 \mathrm{~g} / \mathrm{L}$ glucose and $1 \mathrm{~g} / \mathrm{L}$ peptone. Slants were subcultured biweekly.

Sweet sorghum variety Berény was cultivated at Research Institute, Karcag (Centre for Agricultural Sciences and Engineering, University of Debrecen, Hungary) in 2006. Sweet sorghum juice was extracted from the fresh stem with leaves on by squeezing. Bagasse was collected, chopped and dried at $50^{\circ} \mathrm{C}$ to $85-90 \%$ dry matter content. Before composition analyses it was ground to fine powder.

\section{Steam pretreatment}

Sweet sorghum bagasse was steam pretreated at the Department of Chemical Engineering, Lund University, Sweden. The material was steamed at atmospheric pressure for 1 hour in order to reach an approximately 50\% moisture content, and then impregnated with $2 \% \mathrm{SO}_{2}$ (based on moisture content) in plastic bags for $30 \mathrm{~min}$. Steam pretreatment was performed in a reactor with $10 \mathrm{~L}$ working volume [16]. Temperature was set and maintained by injection of saturated steam. After $10 \mathrm{~min}$ of residence time at $190^{\circ} \mathrm{C}$ the pressure was released and the material was collected in a flash-cyclone. Parameters of steam pretreatment were applied according to Sipos and coworkers [21] who found this combination of temperature and time to be the most effective for increasing cellulose conversion among the studied parameter-combinations

The slurry after pretreatment was collected from the cyclone and washed with triple amount of warm $\left(60-70^{\circ} \mathrm{C}\right)$ distilled water to remove the majority of the water soluble substances, then the liquid and fiber fractions were separated. Therefore, the fraction nominated as "liquid fraction" in present study is a dilution by washing water of the fraction obtained by filter-pressing of the original slurry. The liquid fraction was analyzed for sugar and inhibitor content and the washed fiber fraction was analyzed for structural carbohydrates and lignin content. Both were used as carbon sources in the enzyme production experiments.

\section{Adaptation}

T. reese $i$ was cultured on agar plates containing liquid fraction in different dilutions. This solid medium composed of glucose in $5 \mathrm{~g} / \mathrm{L}$, peptone in $1 \mathrm{~g} / \mathrm{L}$ and agar-agar in $20 \mathrm{~g} / \mathrm{L}$ suspended in either tap water or liquid fraction or mixture of these. $\mathrm{pH}$ was set to 6.0 by adding solid $\mathrm{NaOH}$. The medium was sterilized on $121^{\circ} \mathrm{C}$ for 20 minutes and after that plates were prepared. In the water-liquid fraction mixture the dilution of the liquid fraction was 2 or 4 (1:1 or 1:3 liquid fraction:water ratio).

Plates were inoculated by placing a piece of agar from a previously cultured plate in the middle of the new plate. The plates were grown at room temperature simply in the laboratory. The diameter of the cultures was measured daily in the same time. The rate of growth was expressed as the difference of the areas of the circles covered by the culture on two days and divided by the numbers of days between the two readings $\left(\mathrm{mm}^{2} /\right.$ day). The concentric rings in the conidia formation - caused by the day night cycle of the fungus - made the reading even more exact.

\section{Inoculum preparation}

Conidia from 14-day-old slants were harvested with sterile distilled water. Either this suspension or a piece of the preadapted, T. reesei grown agar plates were used to inoculate Erlenmeyer flasks containing $200 \mathrm{~mL}$ of sterile modified Mandels' medium to obtain a final concentration of $10^{8}$ coni$\mathrm{dia} / \mathrm{mL}$. This medium contained $1.87 \mathrm{~g} / \mathrm{L}\left(\mathrm{NH}_{4}\right)_{2} \mathrm{SO}_{4}, 2.67 \mathrm{~g} / \mathrm{L}$ $\mathrm{KH}_{2} \mathrm{PO}_{4}, 0.53 \mathrm{~g} / \mathrm{L} \mathrm{CaCl}_{2} .2 \mathrm{H}_{2} \mathrm{O}, 0.81 \mathrm{~g} / \mathrm{L} \mathrm{MgSO} \mathrm{M}_{4} .7 \mathrm{H}_{2} \mathrm{O}, 0.40$ $\mathrm{g} / \mathrm{L}$ urea, $5.0 \mathrm{mg} / \mathrm{L} \mathrm{FeSO}_{4} .7 \mathrm{H}_{2} \mathrm{O}, 1.7 \mathrm{mg} / \mathrm{L} \mathrm{MnSO}_{4} . \mathrm{H}_{2} \mathrm{O}, 1.4$ $\mathrm{mg} / \mathrm{L} \mathrm{ZnSO}{ }_{4} .7 \mathrm{H}_{2} \mathrm{O}, 2.0 \mathrm{mg} / \mathrm{L} \mathrm{CoCl} 2.6 \mathrm{H}_{2} \mathrm{O}, 1.00 \mathrm{~g} / \mathrm{L}$ peptone, $0.33 \mathrm{~g} / \mathrm{L}$ yeast extract and $10 \mathrm{~g} / \mathrm{L}$ Solka Floc as carbon source. These components were suspended in either tap water or liquid fraction or a mixture of these in dilutions as indicated later. Inoculated flasks were closed with cotton plugs and incubated at $30^{\circ} \mathrm{C}$ and $300 \mathrm{rpm}$ on a rotary shaker for 4 days.

\section{Shake flask cultivation}

In each case the medium for cellulase production was composed of $\left(\mathrm{NH}_{4}\right)_{2} \mathrm{SO}_{4}$ and $\mathrm{KH}_{2} \mathrm{PO}_{4}$ both in concentration of 0.83 $\mathrm{g} / \mathrm{L}$ and $10 \mathrm{~g} / \mathrm{L}$ washed pretreated bagasse as carbon source. Each medium contained an additional $5 \mathrm{~g} / \mathrm{L}$ wheat distillers' grain as a nitrogen source ( $32.2 \%$ protein) that is also composed of lignocellulosic carbohydrates (18.6\% glucan and $14.6 \%$ xylan). Solid substances were suspended in 0.1 M TRIS-maleic acid buffer ( $\mathrm{pH}$ 5.8) to avoid $\mathrm{pH}$ changes prepared in either tap water or liquid fraction in dilutions indicated later.

Cellulase producing media were inoculated with an aliquot of 4-day-old inoculum at $10 \%(\mathrm{~V} / \mathrm{V})$, and cultures were propagated at $30^{\circ} \mathrm{C}$ and $300 \mathrm{rpm}$ on a rotary shaker. Samples were withdrawn regularly and centrifuged $(3400 \mathrm{~g}, 5 \mathrm{~min})$ to separate supernatants for further analysis. Fermentations were terminated after 9 or 11 days.

\section{Enzyme assay}

Filter Paper Activity (FPA) measurement was carried out according to Mandels et al. [6], with the modification, that an enzyme dilution releasing $1 \mathrm{mg}$ glucose was used. FPA was expressed as FPU/mL, where FPU was defined as the amount of liberated glucose given in micromoles per minute. 


\section{Lignin and carbohydrate analysis}

Lignin and carbohydrate content of raw and pretreated materials were analyzed using NREL protocol [22] with some modification. $0.5 \mathrm{~g}$ of oven dried $\left(105^{\circ} \mathrm{C}\right)$ sample was hydrolyzed with $2.5 \mathrm{ml} 72 \%$ sulfuric acid at room temperature for 2 hours. After that, $75 \mathrm{ml}$ of distilled water was added and the hydrolysis was continued at $121^{\circ} \mathrm{C}$ for $60 \mathrm{~min}$. The samples were filtered through G4 filter crucibles and washed with hot distilled water several times. The remaining lignin on the filter was dried at $105^{\circ} \mathrm{C}$, weighted and placed in furnace at $550^{\circ} \mathrm{C}$ for 6 hours. The Klason lignin content was taken as the ash free residue after hydrolysis.

\section{Sugar analysis}

Reducing sugar contents (RS) during the enzyme fermentation were analyzed using the 2,4-dinitrosalycilic acid reagent as described by Miller [13].

Liquid samples upon their sugar and inhibitor concentrations were analyzed with a Shimadzu HPLC system (Shimadzu, Kyoto, Japan) using an Aminex HPX-87H column (Bio-Rad, Hercules, CA) at $65^{\circ} \mathrm{C}$. The eluent, $5 \mathrm{mM} \mathrm{H}_{2} \mathrm{SO}_{4}$ was used at a flow rate of $0.5 \mathrm{ml} / \mathrm{min}$. Total sugar content (including both monoand oligomers) was determined after mild acid hydrolysis (4\% (V/V) $\mathrm{H}_{2} \mathrm{SO}_{4}, 120^{\circ} \mathrm{C}$ and $30 \mathrm{~min}$ ). All samples were filtered through a $0.2 \mu \mathrm{m}$ pore size filter before HPLC analysis to remove solid particles.

\section{Results and discussion}

\section{Mass balance of the pretreatment}

Fig. 2 shows the mass balance of the pretreatment in terms of hexoses, pentoses and lignin, respectively. The hexose recovery in the fiber fraction was $89 \%$, whereas it was $36 \%$ in case of pentoses suggesting that the hemicelluloses were removed sufficiently. $9 \%$ and $46 \%$ of the hexoses and pentoses, respectively were solubilized, and were present in the liquid fraction after pretreatment. Consequently, the hemicelullose content decreased due to the solubization, while the ratio of lignin and cellulose increased. This represents the aim and the well chosen parameters of the pretreatment, namely the pretreated material contains more and presumably easier accessible cellulose. The composition of the pretreated material is in accordance with the mass balances (Table 1 .

As expected some solubilized sugars, both hexoses and pentoses, were further degraded during the pretreatment to nonsugar compounds. These compounds such as furfural, HMF and aliphatic acids (mostly formic and acetic acid) can inhibit microbial activity under certain circumstances. According to the composition of the separated liquid fraction the carbohydrates, as well as the inhibitors resulted mainly from the hemicellulose (Table 2). The acetic acid and the furfural are decomposition products of hemicellulose. Formic acid arises with the further decomposition of furfural and HMF [15]. The presence of glucose, glucan and HMF shows that due to the severe conditions
Tab. 1. Composition of the washed solid fraction after pretreatment (10 min at $190^{\circ} \mathrm{C}$ ). Mean values of duplicate analyses and standard deviations are presented.

\begin{tabular}{|c|c|}
\hline & $\begin{array}{c}\text { Pretreated bagasse } \\
\text { (separated, washed solid fraction) }\end{array}$ \\
\hline & $\%$ \\
\hline Cellulose & $58.3 \pm 1.2$ \\
\hline Xylan & $12.8 \pm 0.6$ \\
\hline Arabinan & $2.1 \pm 0.1$ \\
\hline Lignin & $24.6 \pm 0.5$ \\
\hline
\end{tabular}

the cellulose has also degraded to some extent in line with the mass balance.

Tab. 2. Composition of the used liquid fraction $\left(10 \mathrm{~min}\right.$ at $\left.190^{\circ} \mathrm{C}\right)$. Mean values of duplicate analyses and standard deviations are presented.

\begin{tabular}{lc}
\hline & $\begin{array}{c}\text { Liquid fraction } \\
\mathrm{g} / \mathrm{L}\end{array}$ \\
\hline Monomers & \\
\hline Glucose & $0.8 \pm 0.0$ \\
Xylose & $3.2 \pm 0.3$ \\
Arabinose & $0.8 \pm 0.1$ \\
\hline Oligomers & \\
\hline Cellobiose & $0.3 \pm 0.0$ \\
Glucan & $1.0 \pm 0.1$ \\
Xylan & $2.3 \pm 0.2$ \\
Arabinan & $0.1 \pm 0.0$ \\
\hline Inhibitors & \\
\hline Acetic acid & $0.7 \pm 0.0$ \\
Formic acid & $0.8 \pm 0.0$ \\
Furfural & $0.4 \pm 0.0$ \\
HMF & $0.1 \pm 0.0$ \\
\hline & \\
\hline
\end{tabular}

\section{Liquid fraction as carbon source for T. reesei}

The inhibitory effect of the liquid fraction and the possibility of adaptation were investigated already in the inoculum phase: $20 \%$ of the water was replaced by liquid fraction, while the parallel reference run contained solely tap water (further referred as reference). It was assumed that in both cases the growth had been initiated based on the dropped $\mathrm{pH}$ values (after 4 days of cultivation). The fall of $\mathrm{pH}$ reflects well the growing of $T$. reesei and it is mainly caused by the consumption of ammonium salts acting as nitrogen source. From the same starting value of $\mathrm{pH}$ 5.70 the final was 3.17 in case of no liquid fraction, while in the broths containing $20 \%$ liquid fraction the $\mathrm{pH}$ was 3.29.

According to Szengyel and Zacchi [24] the sum concentration of furfural and acetic acid as present in the liquid fraction would not lead to growth inhibition. However, this result was obtained in a model medium not in liquid fraction resulting after pretreatment. It is assumed that this difference of inhibition is caused by the other degradation products, mostly from lignin not measured 


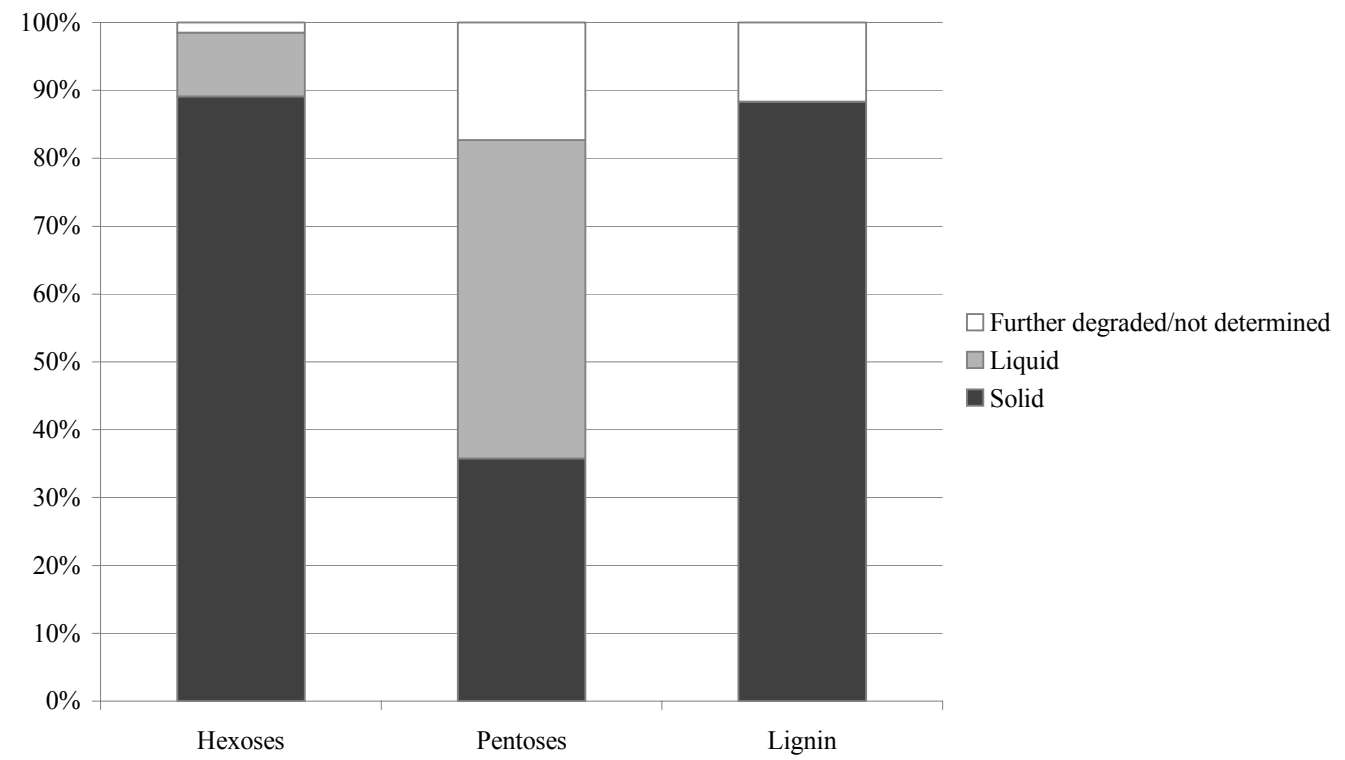

Fig. 2. Mass balance of the pretreatment of sweet sorghum bagasse $\left(10 \mathrm{~min}, 190^{\circ} \mathrm{C}\right)$

here and the synergies between inhibitors.

Both sorts of precultures were used to inoculate medium containing one part of liquid fraction and one part of water (substituted in 50\%). The fermentation was monitored by RS and FPA measurements. Regarding RS the initial 100\% content dropped already in the first 24 hours in both cases. However, there was significant difference between the broths prepared with the different precultures. In case of the broths inoculated with the reference a concentration of $4.6 \mathrm{~g} / \mathrm{L}$ was measured, while in case of the broths prepared with inoculum containing $20 \%$ liquid fraction the RS was $17.3 \%$ lower, $3.8 \mathrm{~g} / \mathrm{L}$. For the 48 th hour this difference nearly disappeared and in both cases the RS zeroed for the 4th day. This profile indicates that the utilization of sugar originating from the liquid fraction is quicker if $T$. reesei had already been adapted already in the inoculum to the liquid fraction.

In FPA profiles similar trends were observed. The difference between the two groups was $12 \%$ observed on day 3 but activities leveled off on day 4 (1.07 FPU/mL in both cases). However, while in the reference broths only minor amount of FPA was produced after day 4 (peaking with $1.27 \mathrm{FPU} / \mathrm{mL}$ at day 9), in the broths initiated with pre-adapted inoculum $1.69 \mathrm{FPU} / \mathrm{mL}$ was reached by day 9 .

The inhibitors usually affect the microbial growth in the initial phase. Therefore it is noteworthy that adaptation of T. reesei can not only lead to quicker initiation of growth but also higher final FPA yield. Due to this significant enhancement of enzyme production in the fermentation experiment, it was assumed that adaptation could be a good strategy to partially overcome the inhibitory effect of the liquid fraction. Therefore, in view of this promising result, our aim was to develop a strain maintenance strategy that results in the utilization of the liquid fraction more effectively.

\section{Pre-adaptation}

Adaptation has been widely investigated in case of yeast strains growing on hydrolysates [18], but the possible adaptation of $T$. reesei has rarely been studied. Bigelow and Wyman [3] reported that adaptation is an encouraging option but because of the difficulty of maintaining the adapted strains no detailed study including enzyme activity measurements was carried out. Hayward and co-workers [10] found that despite adaptation leads to the increased growth, it was not succeed to obtain strains with increased enzyme production. It is noteworthy that the conclusions of both above cited studies came from submerged fermentations.

We aimed to investigate the effect of the liquid fraction on the growth of $T$. reesei in an easy to monitor manner. Therefore $T$. reesei was inoculated on agar plates containing liquid fraction in different dilutions and further subcultured decreasing the dilution of liquid fraction. The reference plates did not contain any liquid fraction. This pre-adaptation was characterized by the diameter of the colonies.

The daily reading of this parameter expresses well the inhibitory nature of the liquid fraction and the ability of the strain to adapt. The measured diameters decreased with increasing ratio of liquid fraction in the media (Table 3). In case of the agar plates prepared with $100 \%$ liquid fraction measurable growth could be observed only in the second week when the colonies on other plates had already overgrown the plates. The changes of cell mass as expressed in diameters is not proportional with the ratio of liquid fraction, for instance on the 5th day the diameter of the culture growing on $25 \%$ and $50 \%$ liquid fraction was $18.6 \%$ and $62.7 \%$ lower, respectively, than the diameter of the reference. Moreover, the difference between the plates was also diminishing over time, on 7 th day it was $9.0 \%$ and $48.7 \%$ respectively.

The adaptation ability expressed in the rate of growth 
Tab. 3. Colony diameters $(\mathrm{mm})$ of $T$. reesei cultures growing on agar plates containing liquid fraction in different dilutions. Mean values of three plates and standard deviations are presented.

\begin{tabular}{lcccc}
\hline Day & Reference & $25 \%$ & $50 \%$ & $100 \%$ \\
\hline 4 & $40 \pm 4.1(100 \%)$ & $29 \pm 3.4(72.5 \%)$ & $12 \pm 2.1(30.0 \%)$ & - \\
5 & $59 \pm 5.3(100 \%)$ & $48 \pm 4.4(81.4 \%)$ & $22 \pm 2.9(37.3 \%)$ & - \\
7 & $78 \pm 5.1(100 \%)$ & $71 \pm 4.4(91.0 \%)$ & $40 \pm 2.9(51.3 \%)$ & - \\
11 & & & & $31 \pm 7.1$ \\
12 & & & & $40 \pm 6.4$ \\
14 & & & $60 \pm 7.1$ \\
\hline
\end{tabular}

$\left(\mathrm{mm}^{2} /\right.$ day $)$ decreased also with the increasing ratio of liquid fraction (Fig. 3). Between day 4 and 5 the growth on the reference plates was the fastest, followed by the plates with liquid fraction indicating the non-linear inhibition with decreasing dilution. In case of 1:3 dilution plates (25\%) after 5 days the rate of growth exceeded those of the reference, showing that the strain was adapted and its growth had no longer been influenced by the presence of liquid fraction in this dilution. On the other hand the decrease of the growing rate on the reference plates may have been caused by carbon limitation as approaching the edge of the plate. In case of 1:1 dilution of the liquid fraction $(50 \%)$, the rate of growth lagged behind those of the reference and the 1:3 dilution. However, over the time the adaptation ability increased as shown by the increasing growth rate.

These results explain well that $T$. reesei can overcome at least partially the inhibition; however, an increasing interval was needed for the adaptation with the increasing liquid fraction ratio. The results also show that successful adaptation does not mean that adapted cultures can compete with colonies growing on non inhibiting media both in terms of growing rate and cell mass as expressed in diameter of colonies.

In order to increase the adaptation-efficiency and approach the features of the cultures growing on the reference plate, a second step was applied. The cultures growing on $25 \%$ and $50 \%$ plates were subcultured to $100 \%$ plates. With this additional step, the growth on the $100 \%$ got measurable on the first week unlike in the first round (Table 4). Moreover, the 50\% $\rightarrow 100 \%$ culture was more successful, showing growth already on the 4th day. These observations proved that the adaptation ability can be maintained and further enhanced by subculturing onto media containing increasing ratio of liquid fraction. However, comparing the diameters to the reference there was still significant difference.

Tab. 4. Colony diameters ( $\mathrm{mm}$ ) of T. reesei cultures growing on agar plates containing liquid fraction in different ratios - second round. Mean values of three plates and standard deviations are presented.

\begin{tabular}{lccc}
\hline Day & Reference & $25 \% \rightarrow 100 \%$ & $50 \% \rightarrow 100 \%$ \\
\hline 4 & $58 \pm 2.1(100 \%)$ & & $19 \pm 0.6(32.8 \%)$ \\
5 & $75 \pm 1.0(100 \%)$ & $4 \pm 0.0(5.3 \%)$ & $31 \pm 1.0(41.3 \%)$ \\
7 & & $11 \pm 0.0$ & $61 \pm 1.0$ \\
\hline
\end{tabular}

With this approach the inhibitory effect of the liquid fraction on the growth of $T$. reesei, as well as the adaptation and the viability can be visualized well (Fig. (4). In the next step these pre-adapted cultures were investigated in shake-flask cellulase fermentation using the pretreated bagasse and other compounds as specified in the Material and methods section suspended in the liquid fraction as additional carbon source.

\section{Enzyme fermentations}

The cultures pre-adapted on agar plates were used to prepare the inocula. However, according to Bigelow and Wyman the conidia do not possess the gained adaptation ability [3], therefore, a piece of agar containing hyphae was cut off from the plates and added into the inoculum media. These media contained the liquid fraction in a ratio agreeing the ratio in the given plate (Table 5). For reference a non-adapted culture of T. reesei was used. After 4 days of cultivation (e.g. prior to inoculating the fermentation medium) the growth of the different inocula was assessed by $\mathrm{pH}$ and FPA. There were no significant differences observed between the broths. In every case the $\mathrm{pH}$ had dropped to near 3.5 and the FPA reached almost $0.5 \mathrm{FPU} / \mathrm{mL}$. This indicates that the adaptation ability was successfully carried from the solid media into the liquid ones.

Tab. 5. Ratio of liquid fraction in liquid Mandels' medium and its agar plate counterpart

\begin{tabular}{lll}
\hline ID number & $\begin{array}{l}\text { Ratio of liquid fraction in } \\
\text { the liquid inoculum }\end{array}$ & $\begin{array}{l}\text { Ratio of liquid fraction in } \\
\text { the respective agar plates }\end{array}$ \\
\hline Nr. 1 & $0 \%$ & $0 \%$ \\
Reference & & \\
\hline Nr. 2 & $25 \%$ & $25 \%$ \\
Nr. 3 & $50 \%$ & $50 \%$ \\
Nr. 4 & $50 \%$ & $25 \% \rightarrow 50 \%$ \\
Nr. 5 & $100 \%$ & $25 \% \rightarrow 100 \%$ \\
Nr. 6 & $100 \%$ & $50 \% \rightarrow 100 \%$ \\
Nr. 7 & $100 \%$ & $100 \%$ \\
\hline
\end{tabular}

The fermentation medium in all cases contained undiluted liquid fraction (in 100\%) besides the other components as given in Materials and methods section. Regarding the RS profile that describes well the fermentation in terms of sugar consumption and so indicating the growth in all cases a slight increase was observed from the initial $9 \mathrm{~g} / \mathrm{L}$ concentration on day 1 (Fig. 5). This is due to the action of cellulase enzymes already present in precultures and carried over at inoculation. While the adapted cultures had already started growing and consuming sugar on day 2 , in reference case it had increased further. The difference could be observed best on day 3 when reference culture started growing and consuming sugar but in smaller extent than the cultures inoculated with adapted $T$. reesei one day before. It could be concluded that the lack of pre-adaptation causes prolongation of lag phase and delays growth as it could be perceived from RS profile of the reference. In case of the pre-adapted broths the RS 


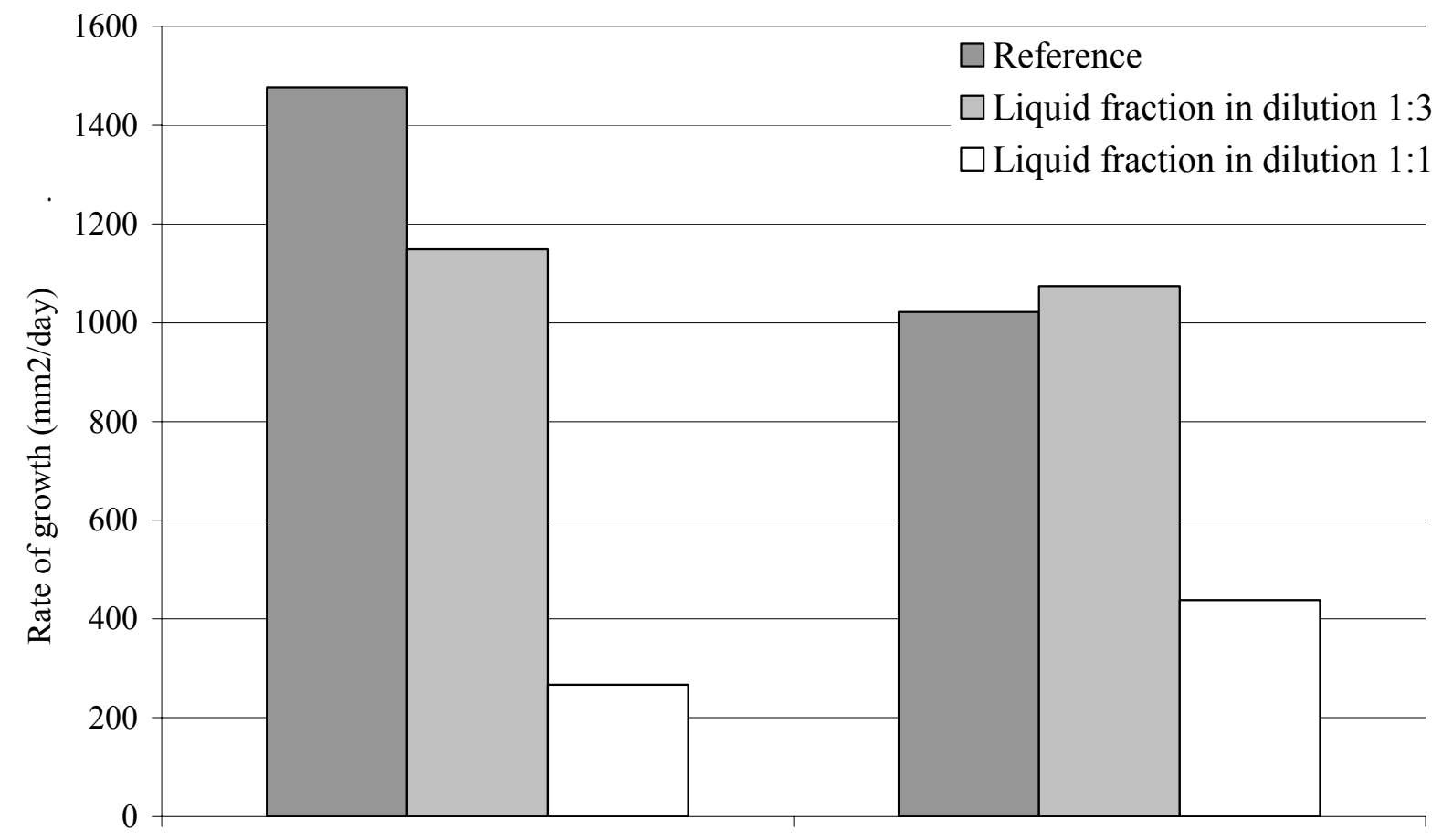

Between day 4 \& 5

Between day 5 \& 7

Fig. 3. Rate of growth of $T$. reesei on plates containing liquid fraction in different dilutions

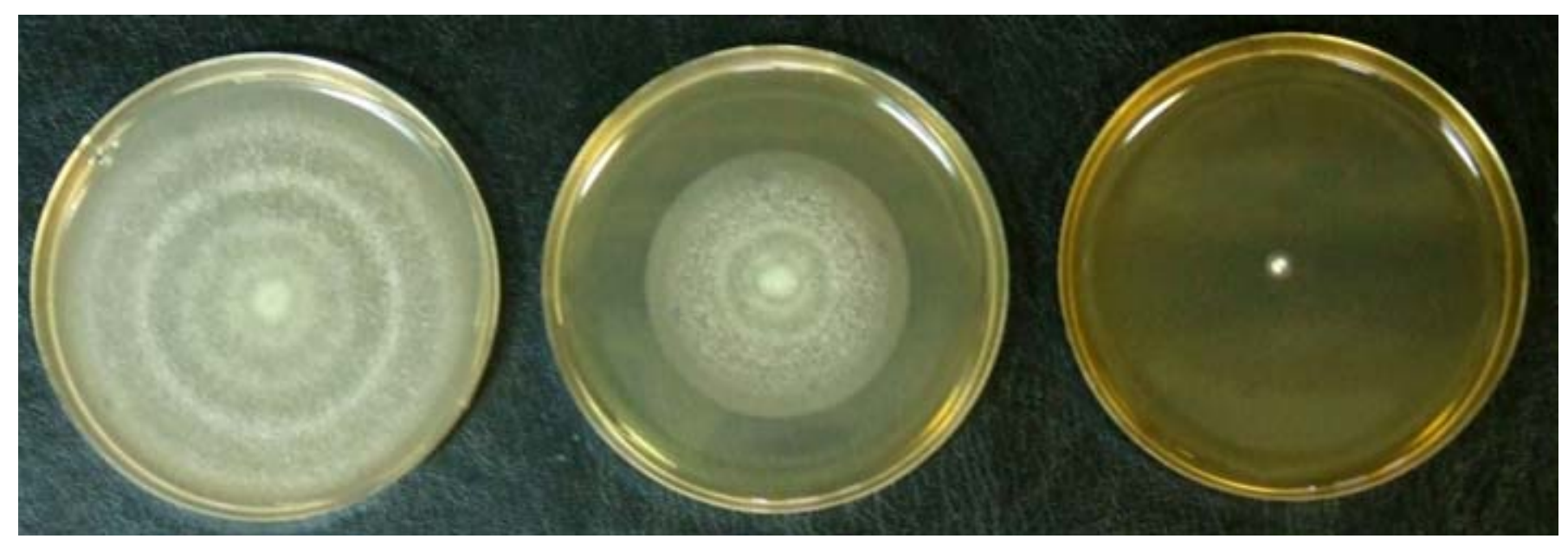

Fig. 4. Images of T. reesei growing on plates containing different ratio of liquid fraction. From the left side: $25 \%, 50 \%, 100 \%, 7$ th day

dropped sharply resulting in an average difference of $77 \%$ between them and the reference. In all cases except the reference RS was equivalent to zero from day 4.

Moreover, not only between adapted broths and reference but also among the adapted broths differences were found. In RS profile a grouping (Nr. $2-\mathrm{Nr} .3 \& 4-\mathrm{Nr} .5,6 \& 7)$ could be observed according to the liquid fraction content of the inoculum and agar plates. The difference from reference was growing with decreasing dilution of the liquid fraction in the inocula: on day 2 in broths of Nr. 2, of the group of Nr. $3 \& 4$ and of the group of Nr. 5, 6, \& 7 the RS content were 5\%, $24 \%$ and $44 \%$ lower, respectively, than in the reference. The observed different patterns in RS consumption and its connection to the liquid fraction ratios in the preculture phase indicate that the final ratio of liquid fraction in the adaptation process is more crucial than in how many steps it had been reached.

The FPA profile also reflects this grouping and the most significant difference was observed on day 3 (Fig. 6). The broths inoculated with either Nr. 5, 6 or 7 (e.g. pre-grown on $100 \%$ liquid fraction agar plates) reached in average $72.3 \%$ higher FPA than the reference. The delayed initiation of growth could be observed here as well. From day 3 the reference culture also started to grow and produce enzyme as reflected on the decreasing differences between FPAs. However, after 11 days the group of Nr. 5, $6 \& 7$ had an average FPA of 1.7 FPU/mL that is $22.6 \%$ higher FPA than those of the reference.

These results are in accordance with the observed growing rates on the plates indicating that with adaptation the longer lag phase caused by the inhibitors can be significantly reduced both in terms of growth and enzyme production. Furthermore 


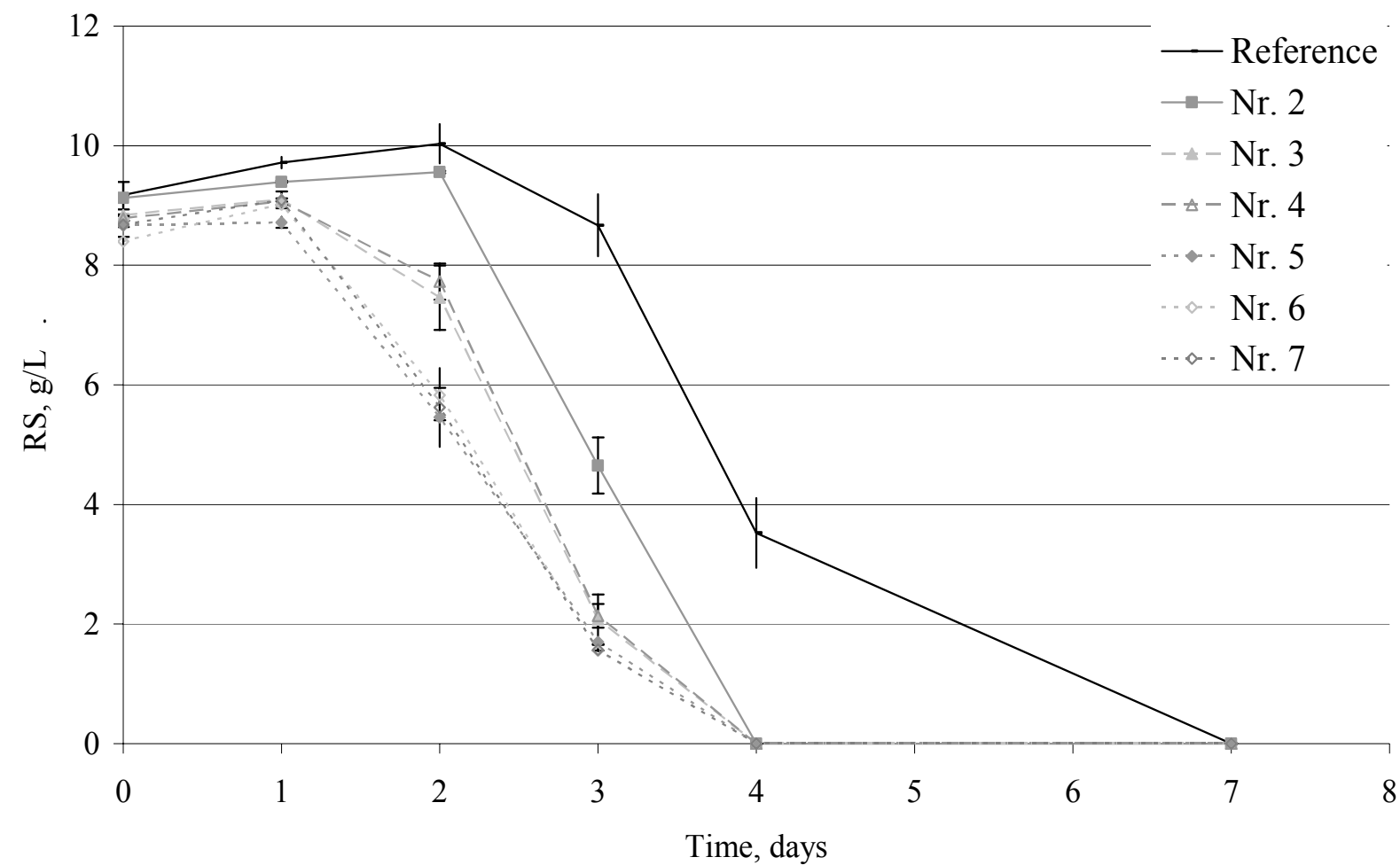

Fig. 5. RS profiles on medium containing undiluted liquid fraction (inoculated with differently pre-adapted $T$. reesei cultures). Numbers refer to the different pre-adaptation conditions on agar plates (percent of liquid fraction in the solid media and in case of multi step adaptation the previous ratio too): Nr. 2 $25 \%$, Nr. $350 \%$, Nr. $425 \% \rightarrow 50 \%$, Nr. $525 \% \rightarrow 100 \%$, Nr. $650 \% \rightarrow 100 \%$, Nr.

and contrarily with the literature stating that no increased enzyme yields can be reached with adaptation [10], in our case the adapted strains achieved also higher final FPA value when growing on liquid fraction resulting also in higher FPA yields, since the carbohydrate content of the broths was the same.

Numbers refer to the different pre-adaptation conditions on agar plates (percent of liquid fraction in the solid media and in case of multi step adaptation the previous ratio too): Nr. 2 $25 \%$, Nr. $350 \%$, Nr. $425 \% \rightarrow 50 \%$, Nr. $525 \% \rightarrow 100 \%$, Nr. 6 $50 \% \rightarrow 100 \%$, Nr. $7100 \%$. The same ratio of liquid fraction as that of the agar plate was applied in each inoculum while fermentations were run in $100 \%$ liquid fraction in all cases. The reference agar plate and inoculum contained no liquid fraction.

\section{Conclusions}

Our results demonstrated that the by-product stream of the pretreatment, called liquid fraction can be used for on-site cellulase production by $T$. reesei despite its inhibitory feature. However, due to its inhibitor content either detoxification is necessary or the strains have to be pre-adapted. By choosing this latter approach we have proved that $T$. reesei can utilize better the liquid fraction in submerged fermentation after a pre-adaptation on solid media. It was found that adaptation could stimulate the initiation of growth and the enzyme production and thus reducing the lag phase. Furthermore, final enzyme activities were found to be higher in case of adapted strains were grown.
$7100 \%$. The same ratio of liquid fraction as that of the agar plate was applied in each inoculum while fermentations were run in $100 \%$ liquid fraction in all cases. The reference agar plate and inoculum contained no liquid fraction. For further information see text and Table 5

\section{References}

1 Almodares A, Hadi MR, Production of bioethanol from sweet sorghum: A review., African Journal of Agricultural Research, 4, (2009), 772-780.

2 Alvira P, Tomás-Pejó E, Ballesteros $\mathbf{M}$, Negro $\mathbf{M} \mathbf{J}$, Pretreatment technologies for an efficient bioethanol production process based on enzymatic hydrolysis: A review, Bioresource Technology, 101, (2010), 4851-4861, DOI 10.1016/j.biortech.2009.11.093.

3 Bigelow M, Wyman C, Cellulase production on bagasse pretreated with hot water, Applied Biochemistry and Biotechnology, 98-100, (2002), 921-934, DOI 10.1385/ABAB:98-100:1-9:921.

4 Chandel AK, da Silva SS, Detoxification of lignocellulosic hydrolysates for improved bioethanol production, Biofuel Production-Recent Developments and Prospects (Marco Aurélio dos Santos Bernardes, ed.)

5 Chandel AK, Chandrasekhar G, Radhika K, Ravinder R, Ravindra P, Bioconversion of pentose sugars into ethanol: A review and future directions, Biotechnology and Molecular Biology Reviews, 6, (2011), 8-20.

6 Eveleigh DE, Mandels M, Andreotti R, Roche C, Measurement of saccharifying cellulase, Biotechnology for Biofuels, 2, (2009), 21, DOI 10.1186/1754-6834-2-21.

7 Gyalai-Korpos M, Feczák J, Réczey K, Sweet sorghum juice and bagasse as a possible feedstock for bioethanol production, Hungarian Journal of Industrial Chemistry Veszprém, 36, (2008n, pages=b43).

8 Gyalai-Korpos M, Mangel R, Alvira P, Dienes D, Ballesteros M, Réczey K, Cellulase production using different streams of wheat grain- and wheat straw-based ethanol processes, Journal of Industrial Microbiology and Biotechnology, 38, (2011), 791-802, DOI 10.1007/s10295-010-0811-9.

9 Hahn-Hägerdal B, Karhumaa K, Fonseca C, Spencer-Martins I, Gorwa-Grauslund MF, Towards industrial pentose-fermenting yeast strains, Applied Microbiology and Biotechnology, 74, (2007), 937-953, DOI 10.1007/s00253-006-0827-2.

10 Hayward T, Hamilton J, Templeton D, Jennings E, Ruth M, Tholudur 


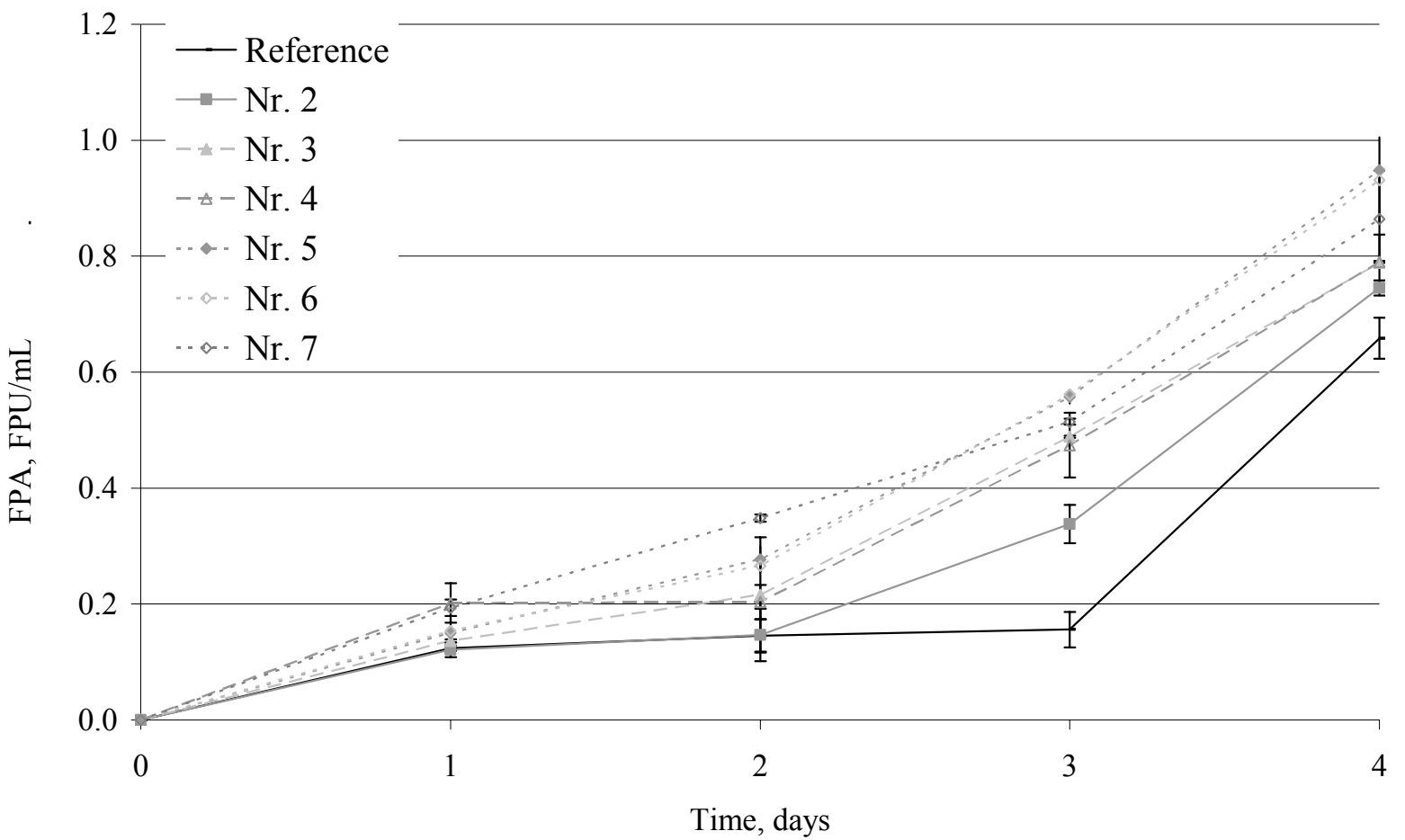

Fig. 6. The initial FPA profiles on undiluted liquid fraction (inoculated with differently adapted $T$. reesei cultures). Numbers refer to the different preadaptation conditions on agar plates (percent of liquid fraction in the solid media and in case of multi step adaptation the previous ratio too): Nr. $225 \%$, Nr. 3 $50 \%$, Nr. $425 \% \rightarrow 50 \%$, Nr. $525 \% \rightarrow 100 \%$, Nr. $650 \% \rightarrow 100 \%$, Nr. $7100 \%$.

A, McMillan J, Tucker M, Mohagheghi A, Enzyme production, growth, and adaptation of T. reesei strains QM9414, L-27, RL-P37, and RUT C-30 to conditioned yellow poplar sawdust hydrolysate., Applied Biochemistry and Biotechnology, 77, (1999), 293-309, DOI 10.1385/ABAB:77:1-3:293.

11 Kálmán G, Réczey K, Possible ways of bio-refining and utilizing the residual lignocelluloses of corn growing and processing, Periodica Polytechnica Chemical Engineering, 51, (2007), 29-36, DOI 10.3311/pp.ch.2007-2.05.

12 Larsson S, Reimann A, Nilvebrant N, Jönsson L, Comparison of different methods for the detoxification of lignocellulose hydrolyzates of spruce, Applied Biochemistry and Biotechnology, 77, (1999), 91-103, DOI 10.1385/ABAB:77:1-3:91.

13 Miller GL, Use of dinitrosalicylic acid reagent for determination of reducing sugar, Analytical Chemistry, 31, (1959), 426-428, DOI 10.1021/ac60147a030.

14 Palmqvist E, Hahn-Hägerdal B, Fermentation of lignocellulosic hydrolysates. I: inhibition and detoxification., Bioresource Technology, 74, (2000), 17-24, DOI 10.1016/S0960-8524(99)00160-1.

15 Palmqvist E, Hahn-Hägerdal B, Fermentation of lignocellulosic hydrolysates. II: inhibitors and mechanisms of inhibition., Bioresource Technology, 74, (2000), 25-33, DOI 10.1016/S0960-8524(99)00161-3.

16 Palmqvist E, Hahn-Hägerdal B, Galbe M, Larsson M, Stenberg K, Szengyel Z, Tengborg C, Zacchi G, Design and operation of a benchscale process development unit for the production of ethanol from lignocellulosics, Bioresource Technology, 58, (1996), 171-179, DOI 10.1016/S09608524(96)00096-X.

17 Palmqvist E, Hahn-Hägerdal B, Szengyel Z, Zacchi G, Réczey K, Simultaneous detoxification and enzyme production of hemicellulose hydrolysates obtained after steam pretreatment, Enzyme and Microbial Technology, 20, (1997), 286-293, DOI 10.1016/S0141-0229(96)00130-5.

18 Parawira W, Tekere M, Biotechnological strategies to overcome inhibitors in lignocellulose hydrolysates for ethanol production: re- 